\title{
Asymptotics of Bivariate Analytic Functions with Algebraic Singularities
}

\author{
Torin Greenwood $\|^{\prime}$ \\ ${ }^{1}$ Georgia Institute of Technology, Atlanta, GA, USA
}

\begin{abstract}
In this paper, we use the multivariate analytic techniques of Pemantle and Wilson to find asymptotic formulae for the coefficients of a broad class of multivariate generating functions with algebraic singularities. Flajolet and Odlyzko (1990) analyzed the coefficients of a class of univariate generating functions with algebraic singularities. These results have been extended to classes of multivariate generating functions by Gao and Richmond (1992) and Hwang $(1996,1998)$, in both cases by immediately reducing the multivariate case to the univariate case. Pemantle and Wilson (2013) outlined new multivariate analytic techniques and used them to analyze the coefficients of rational generating functions.

Résumé. Dans ce papier, on utilise des techniques analytiques multivariées dévéloppées par Pemantle et Wilson pour trouver des développements asymptotiques des coefficients d'une large classe de séries génératrices multivariées avec singularités algébriques. Flajolet et Odlyzko (1990) ont analysé les coefficients d'une classe de séries génératrices univariées avec singularités algébriques. Ces résultats ont été généralisés aux classes de séries génératrices multivariées par Gao et Richmond (1992) et Hwang (1996, 1998). Dans les deux cas, on peut immédiatement réduire le cas multivarié au cas univarié. Pemantle et Wilson (2013) ont décrit de nouvelles techniques analytiques multivariées et ils les ont utilisées pour analyser les coefficients des séries génératrices rationelles.
\end{abstract}

Keywords. generating functions, coefficients, asymptotics, multivariate, singularity analysis, algebraic

\section{Introduction}

Generating functions are a powerful, convenient tool to encode an array of numbers into a single function. Given that a generating function can be computed with limited information about the corresponding array, it is often desirable to learn more about the array from the generating function itself. A particularly useful goal is to approximate the coefficients of a generating function asymptotically as their indices grow.

Researchers have derived asymptotic expansions for the coefficients of many classes of univariate and multivariate generating functions through singularity analysis. In this paper, we will look at the coefficients of $H(x, y)^{-\beta}$, where $H$ is an analytic function and $\beta \notin \mathbb{Z}_{\leq 0}$ is a real number. Under some assumptions about the zero set of $H$, we will find an asymptotic approximation for the coefficients $\left[x^{r} y^{s}\right] H(x, y)^{-\beta}$ as $r$ and $s$ approach infinity with $\frac{r}{s}$ in a nearly-fixed ratio, as described in Theorem 1

\footnotetext{
$\dagger^{\dagger}$ Email: greenwoodemath. gatech.edu. Supported in part by NSF grant DMS-1344199

1365-8050 (c) 2016 Discrete Mathematics and Theoretical Computer Science (DMTCS), Nancy, France
} 
In 1990, Flajolet and Odlyzko found asymptotics for a large class of univariate functions with algebraic singularities by using the Cauchy integral formula and explicit contour manipulations. When researchers first extended these results to classes of bivariate functions, they relied on temporarily fixing a variable and applying univariate results, which required special restrictions on the bivariate functions. In this paper, we instead rely on the multivariate techniques that Pemantle and Wilson developed in [PW13] and their previous work. Pemantle and Wilson begin with the multivariate Cauchy integral formula and manipulate it directly. More details are in Section 2 below.

Finally, in Section 7, we examine an application of Theorem 1 to a generating function that encodes properties of random colorings on the complete graph, as found in [BCCG15].

\section{Multivariate Analytic Combinatorics of Rational Functions}

In [PW13], Pemantle and Wilson outline a program which greatly extends the results of previous work on multivariate generating function analysis. In the simplest case, they begin with a rational function, $F(\mathbf{z})=G(\mathbf{z}) / H(\mathbf{z})$, where $G$ and $H$ are polynomials with real coefficients in the variables $z_{1}, \ldots, z_{d}$, and where $F(\mathbf{z})$ is analytic near the origin. We will write $\mathbf{z}=\left(z_{1}, \ldots, z_{d}\right)$ and $\mathbf{z}^{\mathbf{r}}=z_{1}^{r_{1}} \cdots z_{d}^{r_{d}}$. Then, $F(\mathbf{z})$ has the series representation:

$$
F(\mathbf{z})=\sum_{\mathbf{r} \in \mathbb{N}^{d}} a_{\mathbf{r}} \mathbf{z}^{\mathbf{r}}
$$

The multivariate Cauchy integral formula tells us:

$$
\left[\mathbf{z}^{\mathbf{r}}\right] F(\mathbf{z})=\left(\frac{1}{2 \pi i}\right)^{d} \int_{T} F(\mathbf{z}) \mathbf{z}^{-\mathbf{r}-1} \mathrm{~d} \mathbf{z}
$$

The torus $T=\left\{\left|z_{1}\right|=c_{1}\right\} \times \cdots \times\left\{\left|z_{d}\right|=c_{d}\right\}$ is small enough that it does not enclose any singularities of $F(\mathbf{z})$. The goal is to approximate $\left[\mathbf{z}^{n \hat{\mathbf{r}}}\right] F(\mathbf{z})$ for some fixed unit vector $\hat{\mathbf{r}} \in \mathbb{R}_{\geq 0}^{d}$ as $n$ approaches infinity.

To analyze the Cauchy integral, we will expand the torus $T$ into a cycle $\mathcal{C}$ which gets stuck on some chosen subset of the singularities of $F(\mathbf{z})$ (which are the zeroes of $H(\mathbf{z})$ ), and expands beyond them elsewhere. Due to the $\mathbf{z}^{-\mathbf{r}}$ term in the integrand, we hope that as $\mathbf{r} \rightarrow \infty$, the integrand will decay exponentially faster in the regions of $\mathcal{C}$ away from the singularities of $F$, since the magnitude of $\mathbf{z}$ is larger in these regions. If this is true, then we can approximate the integral by analyzing the integrand near the singularities, since the rest of the integral decays too quickly to contribute to the asymptotics. However, we will need to choose our expansion of $T$ carefully in order to ensure this works.

We will aim to minimize the maximum modulus of $\mathbf{z}^{-\mathbf{r}}$ along our contour $\mathcal{C}$. The reason for this is as follows: we want to find a contour where the integrand attains its maximum modulus over some small interval, and then decays rapidly away from this interval. At a point where the maximum modulus is not minimized, the argument of the $\mathbf{z}^{-\mathbf{r}}$ term will oscillate rapidly as $\mathbf{r}$ tends to infinity, which leads to cancellation near the singularity. However, when the maximum modulus is minimized, we will be able to approximate the integral in this region by using saddle point methods.

To minimize the maximum modulus, we will consider the height function, $h(\mathbf{z}):=-\hat{\mathbf{r}} \cdot \operatorname{Re} \log \mathbf{z}$. Although this excludes the contribution from $F(\mathbf{z})$ in the integrand, $F(\mathbf{z})$ is bounded on compact sets, so $h$ still approximates the $\log$ modulus of the integrand as $\mathbf{r}$ approaches infinity in the direction of $\hat{\mathbf{r}}$. Now, knowing that we will expand the torus $T$ until it hits a singularity of $F$, we will consider the values of $h$ on 
$\mathcal{V}:=\{\mathbf{z}: H(\mathbf{z})=0\}$. On a cycle where the maximum of $h$ is minimized, the points where the maximum of $h$ is attained are saddle points of $h$. Thus, the critical points of $h$ restricted to $\mathcal{V}$ will be candidates for the singularities that will contribute to the asymptotics.

To find the critical points of $h$, we consider a stratification of the space $\mathcal{V}$, and we restrict our attention to critical points within a certain stratum $S$. When $\mathcal{V}$ is a smooth manifold near a critical point, the critical point is called smooth. In this case, the stratum $S$ is of dimension $d-1$, and we can find $d-1$ equations (in addition to $H=0$ ) that characterize the location of the smooth critical points:

$$
r_{1} z_{2} \frac{\partial H}{\partial z_{2}}=r_{2} z_{1} \frac{\partial H}{\partial z_{1}}, \ldots, r_{1} z_{d} \frac{\partial H}{\partial z_{d}}=r_{d} z_{1} \frac{\partial H}{\partial z_{1}}
$$

In general, it is not necessarily true that all critical points will contribute to the leading term of the Cauchy integral, but we will require that the critical points be minimal (described in Section 3 below), which will guarantee that they do.

After determining which critical points are candidates for contributing to the asymptotics, we still must expand the torus $T$ into a cycle $\mathcal{C}$ which hugs $\mathcal{V}$ near these points. In the general case, this cycle can be described using Morse theory. Here, we will rely on explicit contour deformations to define $\mathcal{C}$. The portion of the contour near a particular critical point is called a quasi-local cycle. The asymptotics of the coefficients are thus given by a sum of integrals over these quasi-local cycles. For rational generating functions, the integrals over quasi-local cycles can be computed by using residues. Beyond just finding leading-term asymptotics, these analyses can be used to find complete asymptotic expansions of coefficients, as in Raichev and Wilson's work in [RW08].

\section{Bivariate Analytic Functions with Algebraic Singularities}

Our goal is to find the asymptotics of the coefficients of $H(x, y)^{-\beta}$, where $H$ is an analytic function with real coefficients and $\beta \in \mathbb{R}$ is not a negative integer. Let us summarize notation in a bivariate setting. Let $\mathcal{V}$ be the zero set of the analytic function, $H(x, y)$, where $H(0,0) \neq 0$. We will approximate the coefficients $\left[x^{r} y^{s}\right] H(x, y)^{-\beta}$ for a fixed $\beta \in \mathbb{R}$ as $r$ and $s$ approach infinity with their ratio approaching

a constant, $\lambda$. Critical points in the direction of $\lambda=\frac{r+O(1)}{s}$ (as $r$ and $s$ approach infinity) are defined by:

$$
\begin{aligned}
H & =0 \\
r y \frac{\partial H}{\partial y} & =s x \frac{\partial H}{\partial x}
\end{aligned}
$$

The critical points are smooth if the gradient of $H$ does not vanish on $\mathcal{V}$ at the critical points. Let $\mathcal{D}$ be the domain of convergence of the power series of $H^{-\beta}$ that converges around the origin, $(0,0)$. Then, a critical point $(p, q)$ is called minimal if $(p, q) \in \partial \mathcal{D}$. We will apply heuristics from Section 2 to prove the following:

Theorem 1 Let $H$ be an analytic function with exactly $n$ strictly minimal critical points $\left\{\left(p_{i}, q_{i}\right)\right\}_{i=1}^{n}$, all of which are smooth and lie on the same torus $T^{*}$. (Hence, $\left|p_{i}\right|=\left|p_{j}\right|$ and $\left|q_{i}\right|=\left|q_{j}\right|$ for all $1 \leq i, j \leq n$.) Let $\beta \in \mathbb{R}$ with $\beta \notin \mathbb{Z}_{\leq 0}$, and let $\lambda=\frac{r+O(1)}{s}$ as $r, s \rightarrow \infty$. Define $\chi_{1}, \chi_{2}$, and $M_{i}$ as follows (where $\chi_{1}$ 
and $\chi_{2}$ depend on i):

$$
\begin{aligned}
\chi_{1} & =\frac{H_{y}\left(p_{i}, q_{i}\right)}{H_{x}\left(p_{i}, q_{i}\right)}=\frac{p_{i}}{\lambda q_{i}} \\
\chi_{2} & =\left.\frac{1}{2 H_{x}}\left(\chi_{1}^{2} H_{x x}-2 \chi_{1} H_{x y}+H_{y y}\right)\right|_{(x, y)=\left(p_{i}, q_{i}\right)} \\
M_{i} & =-\frac{2 \chi_{2}}{p_{i}}-\frac{\chi_{1}^{2}}{p_{i}^{2}}-\frac{1}{\lambda q_{i}^{2}}
\end{aligned}
$$

For all $i$, assume $p_{i}, q_{i}, H_{x}\left(p_{i}, q_{i}\right)$, and $M_{i}$ are nonzero, and assume that the real part of $-q^{2} M$ is strictly positive. Define $\left\{x^{-\beta}\right\}_{P}$ as the value of $x^{-\beta}$ defined by using a ray from the origin of $\mathbb{C}$ as the branch cut of the logarithm. In this definition, choose any ray such that $\left\{H(x, y)^{-\beta}\right\}_{P}=H(x, y)^{-\beta}$ in a neighborhood of the origin in $\mathbb{C}^{2}$ (as defined by the power series of $H^{-\beta}$ ), and such that this ray does not pass through $-p_{i} H_{x}\left(p_{i}, q_{i}\right)$ for any $i$. Let $\omega_{i}$ be the signed number of times the curve $H\left(t p_{i}, t q_{i}\right)$ crosses this branch cut in a counterclockwise direction as $t$ increases, $0 \leq t<1$. Then, the following expression holds as $r, s \rightarrow \infty$ :

$$
\left[x^{r} y^{s}\right] H(x, y)^{-\beta}=\sum_{i=1}^{n} \frac{r^{\beta-\frac{3}{2}} p_{i}^{-r} q_{i}^{-s}\left\{\left(-H_{x}\left(p_{i}, q_{i}\right) p_{i}\right)^{-\beta}\right\}_{P} e^{-\beta\left(2 \pi i \omega_{i}\right)}}{\Gamma(\beta) \sqrt{-2 \pi q_{i}^{2} M_{i}}}+o\left(r^{\beta-\frac{3}{2}} p_{1}^{-r} q_{1}^{-s}\right)
$$

Here, the square root in the denominator is taken to be the principal root.

Unfortunately, for general $H$, the formula in Theorem 1 is messy, as we must find how many times the image of $H$ wraps around the origin along the path connecting $(0,0)$ to each critical point $(p, q)$, and it is difficult to determine the sign of the square root. Luckily, in the case where $H$ has only real coefficients and there is a single smooth strictly minimal critical point, we can simplify the formula.

Corollary 1 Let $H$ be an analytic function with a single smooth strictly minimal critical point $(p, q)$, where $p$ and $q$ are real and positive. Let $H$ have only real coefficients in its power series expansion about the origin. Assume $H(0,0)>0$, and consider $H^{-\beta}$ for $\beta \in \mathbb{R}$ with $\beta \notin \mathbb{Z}_{\leq 0}$. Also, define $H^{-\beta}$ here with the standard branch chosen along the negative real axis, so that $H(0,0)^{-\beta}>0$. Let $\lambda=\frac{r+O(1)}{s}$ as $r, s \rightarrow \infty$. Define the following quantities:

$$
\begin{aligned}
\chi_{1} & =\frac{H_{y}(p, q)}{H_{x}(p, q)}=\frac{p}{\lambda q} \\
\chi_{2} & =\left.\frac{1}{2 H_{x}}\left(\chi_{1}^{2} H_{x x}-2 \chi_{1} H_{x y}+H_{y y}\right)\right|_{(x, y)=(p, q)} \\
M & =-\frac{2 \chi_{2}}{p}-\frac{\chi_{1}^{2}}{p^{2}}-\frac{1}{\lambda q^{2}}
\end{aligned}
$$

Assume that $H_{x}(p, q)$ and $M$ are nonzero. Then, the following expression holds as $r, s \rightarrow \infty$ :

$$
\left[x^{r} y^{s}\right] H(x, y)^{-\beta} \sim \frac{r^{\beta-\frac{3}{2}} p^{-r} q^{-s}\left(-H_{x}(p, q) p\right)^{-\beta}}{\Gamma(\beta) \sqrt{-2 \pi q^{2} M}}
$$

In the above expression, $-H_{x}(p, q) p$ will be a positive real number, and $\left(-H_{x}(p, q) p\right)^{-\beta}$ will also be a positive real number. Additionally, $-2 \pi q^{2} M$ is positive, so the positive square root is taken. 


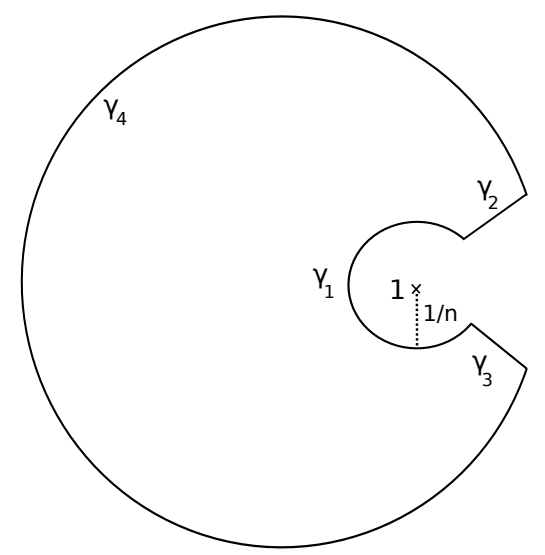

Fig. 1: The expanded contour, $\mathcal{C}^{*}$, used in Flajolet and Odlyzko's proof.

\subsection{Historical Background}

In their 1990 paper [FO90], Flajolet and Odlyzko described how to compute the asymptotics of a class of univariate generating functions with algebraic singularities. They considered functions of the form,

$$
g(z)=K(1-z)^{\alpha}(\log (1-z))^{\gamma}(\log \log (1-z))^{\delta},
$$

where $\alpha, \gamma, \delta$, and $K$ are arbitrary real numbers, along with other related classes of functions. Their results differed from previous results both in the class of generating functions covered, and in their method of proof. Because we will use similar techniques in our proofs later, we take a moment to summarize their proof here. Flajolet and Odlyzko relied on the univariate Cauchy integral formula:

$$
\left[z^{n}\right] g(z)=\frac{1}{2 \pi i} \int_{\mathcal{C}} g(z) \frac{d z}{z^{n+1}}
$$

Here, $\left[z^{n}\right] g(z)$ represents the coefficient of $z^{n}$ in the power series expansion of $g$, and $\mathcal{C}$ is any positivelyoriented contour around the origin which does not enclose any singularities of $g(z)$. Starting with any function $f$ such that $f(z)=O\left(|1-z|^{\alpha}\right)$ as $z \rightarrow 1$, and letting $\mathcal{C}$ be a small circle around the origin, the authors expanded $\mathcal{C}$ in hopes of finding a contour which is easier to analyze. As $\mathcal{C}$ expands, it must avoid not only the singularity at 1 , but also the branch cut emanating from this point. They expand the contour so it looks like a Hankel contour, $\mathcal{C}^{*}$, as shown in Figure 1 . Once expanded, they analyzed the integral along each part of the contour, $\gamma_{i}$, separately.

Later in the 1990s, other researchers extended these results to classes of multivariate generating functions. Bender and Richmond, [BR83], had already considered the asymptotics of multivariate generating functions with poles in 1983. In 1992, Gao and Richmond, [GR92], considered classes of bivariate generating functions $F(z, x)$ which are of a form they called algebraico-logarithmic, which includes some generating functions with algebraic singularities. These algebraico-logarithmic functions could be reduced to a univariate generating function where the results of Flajolet and Odlyzko can be applied. Then, in his 1996 and 1998 papers, [Hwa96] and [Hwa98], Hwang expanded upon the multivariate results, using a probability framework and deriving large deviation theorems. 


\section{Proof Set-Up}

To prove Theorem 1, we analyze the multivariate Cauchy integral formula, (1). An outline of the analysis is as follows: we find a change of variables into $(u, v)$ coordinates so that the analytic function $H(x, y)$ behaves essentially as a linear function in $u$, with some minor error terms in $v$. Then, we describe an appropriate expansion of the torus $T$ in the Cauchy integral formula. With this contour, we will justify approximating the resulting integral by an iterated integral, relying on the fact that $H$ is nearly linear. This step is by far the most tedious, and takes many lemmas to justify. Finally, we analyze this iterated integral.

\subsection{A Convenient Change of Variables}

In order to approximate $H(x, y)$ as a univariate linear function near the critical point $(p, q)$, it turns out it is sufficient that the power series expansion of $H$ has no constant term, linear term, nor quadratic term in one of its two input variables. To transform $H$ into this form, we define the following change of variables:

$$
\begin{aligned}
& u=x+\chi_{1}(y-q)+\chi_{2}(y-q)^{2} \\
& v=y
\end{aligned}
$$

Here, $\chi_{1}$ and $\chi_{2}$ are as defined in Theorem 1 above. Write $H$ as a power series in $u$ and $v$ :

$$
H(x, y)=\sum_{m, n \geq 0} d_{m n}(u-p)^{m}(v-q)^{n}=: \tilde{H}(u, v)
$$

Since $H(p, q)=0$, we have that $d_{00}=0$. Notice that when $(x, y)=(p, q)$, we also have that $(u, v)=$ $(p, q)$. We can easily verify that $d_{01}=d_{02}=0$ by checking some derivatives of $H$.

\subsection{Determining the Quasi-Local Cycle}

For now, assume that there is a unique critical point, $(p, q)$. Recall that the original domain of integration in (1) is a torus $T$ around the origin which encloses no singularities of $H^{-\beta}(x, y)$. To decrease the magnitude of the integrand exponentially as $r$ and $s$ approach infinity, we will expand the torus $T$ towards the minimal critical point, $(p, q)$. Because $(p, q)$ is a strictly minimal critical point, there cannot be any zeroes between the origin and $(p, q)$ that would otherwise obstruct the deformation. Hence, we can expand the domain of integration through a homotopy until it is near the critical point.

Before expanding the torus, $T$ is the product of a small $x$ circle and a small $y$ circle. Begin the deformation by expanding the $y$ component to the circle, $|y|=|q|$. The $y$ portion of the quasi-local cycle, $\mathcal{C}_{y}$, will be the part of this circle where $y=q e^{i \theta}$ for $|\theta| \leq \theta_{y}$, where $\theta_{y}>0$ is a small constant. Note that $q$ is not necessarily real. This contour is pictured on the left in Figure 2 .

Now, for each $y \in \mathcal{C}_{y}$, we will expand the $x$ circle until it approaches the zero set of $H$ near $p$. When $y$ is close to $q$, we will wrap the $x$ contour around the zero set of $H$. However, when $y$ is further away from $q$, we will expand the $x$ contour less, so that it does not come into contact with the zero set of $H$.

More explicitly, since $H_{x}(p, q) \neq 0$ and $H$ is analytic, the implicit function theorem guarantees that we can parameterize the variety $\mathcal{V}=\{(x, y) \mid H(x, y)=0\}$ by a smooth function $G(y)$, so that $H(p+$ $G(y), y)=0$ for all $y \in \mathcal{C}_{y}$ with $\theta_{y}$ sufficiently small. So, for $y=q e^{i \theta}$ with $|\theta| \leq \frac{\theta_{y}}{2}$, we choose the $x$ contour appearing on the right in Figure 2 It is not necessarily true that this contour avoids the branch cut of $H^{-\beta}$ : to account for this, we can view all computations as if they are on the Riemann surface of 

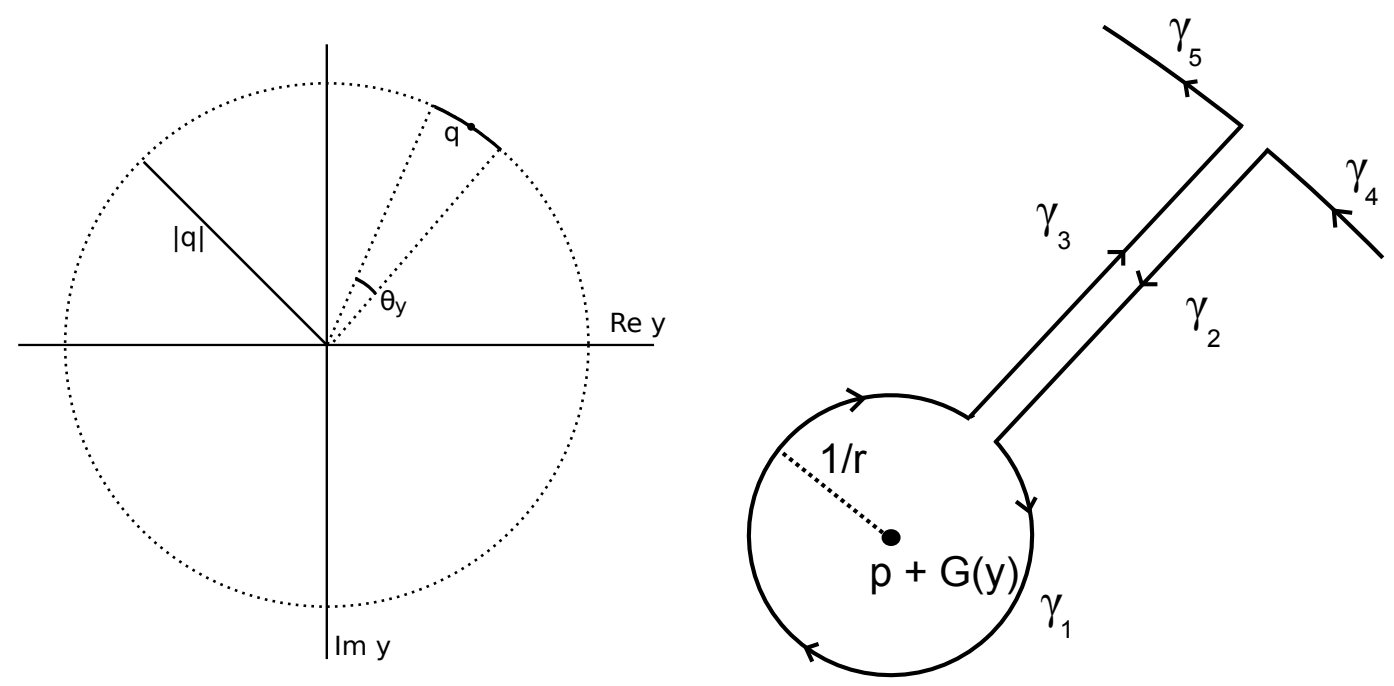

Fig. 2: On the left, the $y$ portion of the quasi-local contour. On the right, a close-up of the $x$ portion of the quasi-local contour.

$H^{-\beta}$, and then readjust our arguments accordingly when analyzing the Cauchy integral. Note that as $|\theta|$ increases with $|\theta| \geq \frac{\theta_{y}}{2}$, we can find an interpolation of the $x$ quasi-local contour, shrinking it until it no longer wraps around the zero set of $H$. Additionally, the change of variables into $(u, v)$ coordinates will allow us to drop the corresponding $G(y)$ term in the contour when sufficiently close to the critical point $(p, q)$.

In summary, the final quasi-local cycle $\mathcal{C}(p, q)$ (in $(u, v)$-coordinates) near the critical point $(p, q)$ has three regimes. The contour is an arc in $v$, and wraps around the zero set of $\tilde{H}$ in $u$. Let $v=q e^{i \theta}$. When $\theta \leq r^{-\frac{2}{5}}$, the $u$-contour wraps exactly around the point, $p$, and this portion of the contour is a product contour. When $r^{-\frac{2}{5}} \leq \theta \leq \frac{\theta_{y}}{2}$, the contour instead wraps around the point $p+\tilde{\kappa}(v)$ for a suitably chosen interpolation $\tilde{\kappa}$. Finally, if $\theta \geq \frac{\theta_{y}}{2}$, then the $u$-contour gradually shrinks as $\theta$ increases, until it no longer intersects the zero set of $\tilde{H}$ at all.

With this explicit quasi-local contour, we must also justify that the integral decays rapidly away from the quasi-local contour. This can be done by carefully defining how to expand the torus so that each point on the contour $\mathcal{C}$ has a large magnitude away from $(p, q)$, forcing the $\mathbf{z}^{-\mathbf{r}}$ term to decay exponentially faster along this region when compared to along the quasi-local contour itself.

\section{Approximating with a Product Integral}

After applying the change of variables to the Cauchy integral formula (1) restricted to the quasi-local cycle near $(p, q)$, written $\mathcal{C}(p, q)$, we obtain the following integral:

$$
\left(\frac{1}{2 \pi i}\right)^{2} \iint_{\mathcal{C}(p, q)} \tilde{H}(u, v)^{-\beta}\left(u-\chi_{1}(v-q)-\chi_{2}(v-q)^{2}\right)^{-r-1} v^{-s-1} \mathrm{~d} u \mathrm{~d} v
$$


Here, we used the fact that the Jacobian of the transformation is 1 . Our goal now is to show that this integral is essentially a product integral. The following lemma describes this precisely.

\section{Lemma 1}

$$
\begin{aligned}
& \left(\frac{1}{2 \pi i}\right)^{2} \iint_{\mathcal{C}(p, q)} \tilde{H}(u, v)^{-\beta}\left(u-\chi_{1}(v-q)-\chi_{2}(v-q)^{2}\right)^{-r-1} v^{-s-1} \mathrm{~d} u \mathrm{~d} v \\
& \sim\left(\frac{1}{2 \pi i}\right)^{2} \iint_{\mathcal{C}_{\ell}(p, q)}\left[H_{x}(p, q) \cdot(u-p)\right]^{-\beta} u^{-r-1} v^{-s-1}\left[1-\frac{\chi_{1}(v-q)+\chi_{2}(v-q)^{2}}{p}\right]^{-r-1} \mathrm{~d} u \mathrm{~d} v
\end{aligned}
$$

The above estimate holds as $r, s \rightarrow \infty$ with $\lambda=\frac{r+O(1)}{s}$. Here, $\mathcal{C}_{\ell}(p, q)$ is the portion of $\mathcal{C}(p, q)$ where $|\theta| \leq r^{-\frac{2}{5}}$. Hence, $\mathcal{C}_{\ell}(p, q)$ is a product contour.

The proof of this lemma involves two types of statements: near the critical point, where $|u-p|$ and $|v-q|$ are both sufficiently small, we will argue that the integrands are asymptotically the same. Away from the critical point, where at least one of $|u-p|$ or $|v-q|$ is sufficiently large, we will show that both integrands are small, and hence do not contribute asymptotically to either integral. (In the second integral, we need only show that the integrand is small when $|u-p|$ is large, since $|v-q|$ is always small in $\mathcal{C}_{\ell}(p, q)$.)

In order to match the two integrands when $|v-q|$ and $|u-p|$ are small, we rewrite the original:

$$
\begin{aligned}
\tilde{H}(u, v)^{-\beta}\left(u-\chi_{1}(v-q)-\chi_{2}(v-q)^{2}\right)^{-r-1} v^{-s-1} \\
\quad=\left[H_{x}(p, q) \cdot(u-p)\right]^{-\beta} u^{-r-1} v^{-s-1}\left[1-\frac{\chi_{1}(v-q)+\chi_{2}(v-q)^{2}}{p}\right]^{-r-1} K(u, v) L(u, v)
\end{aligned}
$$

Here, $K$ and $L$ are correction factors with the following definitions:

$$
K(u, v):=\left(\frac{1-\frac{\chi_{1}(v-q)+\chi_{2}(v-q)^{2}}{u}}{1-\frac{\chi_{1}(v-q)+\chi_{2}(v-q)^{2}}{p}}\right)^{r-1}, \quad L(u, v):=\left[\frac{\tilde{H}(u, v)}{H_{x}(p, q)(u-p)}\right]^{-\beta}
$$

Thus, our goal is to show that $K$ and $L$ are asymptotically equal to 1 . We can analyze $K$ and $L$ along each part of the contour where $(u-p)$ and $(v-q)$ are small, and verify that in every case, they are asymptotically equal to $1+o(1)$ as $r, s \rightarrow \infty$ with $\lambda=\frac{r+O(1)}{s}$. These details are technical but not challenging, and will appear in the full version of the paper.

Away from the critical point, the contribution to the integral decays exponentially faster than the contribution near the critical point. We show this by comparing our final approximation for the coefficients of $H^{-\beta}$ to the magnitude of the integrand on the portion of the contour away from $(p, q)$.

\section{Analyzing the Product Integral}

Lemma 1 has reduced our work to computing the following:

$$
\left(\frac{1}{2 \pi i}\right)^{2} \iint_{\mathcal{C}_{\ell}(p, q)}\left[H_{x}(p, q) \cdot(u-p)\right]^{-\beta} u^{-r-1} v^{-s-1}\left[1-\frac{\chi_{1}(v-q)+\chi_{2}(v-q)^{2}}{p}\right]^{-r-1} \mathrm{~d} u \mathrm{~d} v
$$




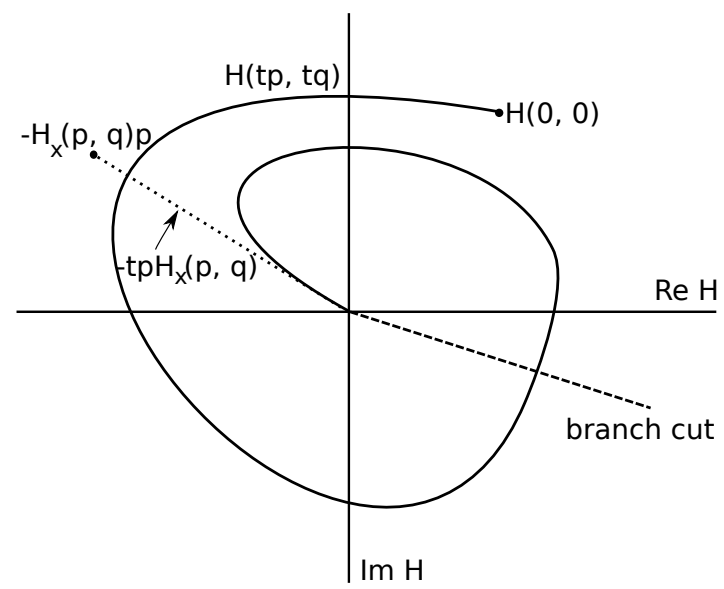

Fig. 3: An example with $\omega=1$.

We break it up into two univariate integrals, to be analyzed separately:

$$
\left(\frac{1}{2 \pi i}\right)^{2}\left(\int_{U}\left[H_{x}(p, q) \cdot(u-p)\right]^{-\beta} u^{-r-1} \mathrm{~d} u\right)\left(\int_{V} v^{-s-1}\left[1-\frac{\chi_{1}(v-q)+\chi_{2}(v-q)^{2}}{p}\right]^{-r-1} \mathrm{~d} v\right)
$$

Above, $U$ is the $u$-projection of the contour, $\mathcal{C}_{\ell}$, which resembles the $x$ contour in Figure 2 , but with $G(y)=0 . V$ is likewise the $v$-projection, which is the set, $\left\{v: v=q e^{i \theta},|\theta| \leq r^{-\frac{2}{5}}\right\}$. These integrals can be analyzed separately.

Lemma 2 The following holds uniformly as $r, s \rightarrow \infty$ with $\lambda=\frac{r+O(1)}{s}$ :

$$
\int_{U}\left[H_{x}(p, q) \cdot(u-p)\right]^{-\beta} u^{-r-1} \mathrm{~d} u=\frac{2 \pi i}{\Gamma(\beta)} r^{\beta-1} p^{-r}\left\{\left(-H_{x}(p, q) p\right)^{-\beta}\right\}_{P} e^{-\beta(2 \pi i \omega)}+o\left(r^{\beta-1} p^{-r}\right)
$$

Here, $\omega$ is defined to be the signed number of times the curve $H(t p, t q)$ crosses the branch cut in the definition of the function $\left\{x^{-\beta}\right\}_{P}$, as described in the statement of the Theorem.

The main idea behind the proof of this lemma is that the remaining integral is almost a univariate Cauchy integral, but with a shrunken domain of integration. Thus, the integral is approximately equal to the coefficients of $\left[H_{x}(p, q) \cdot(u-p)\right]^{-\beta}$, which can be estimated using the binomial theorem and Stirling's approximation. To account for the branch cut in the original function $H(x, y)^{-\beta}$, we add a term $e^{-\beta(2 \pi i \omega)}$, where $\omega$ counts the number of times the image of $H$ wraps around the origin as the input increases from $(0,0)$ to $(p, q)$. An example is illustrated in Figure 3

We turn our attention to the other integral, and find its asymptotic contribution. 
Lemma 3 The following holds uniformly as $r, s \rightarrow \infty$ with $\lambda=\frac{r+O(1)}{s}$ :

$$
\int_{V} v^{-s-1}\left[1-\frac{\chi_{1}(v-q)+\chi_{2}(v-q)^{2}}{p}\right]^{-r-1} \mathrm{~d} v=i q^{-s} \sqrt{\frac{2 \pi}{-q^{2} M r}}+o\left(q^{-s} r^{-\frac{1}{2}}\right)
$$

Here, the square root is taken to be the principal root.

This integral is nearly a Fourier-Laplace integral, again with a shrunken domain of integration, and thus can be estimated using standard Fourier-Laplace approximations. Multiplying the approximations from these two lemmas yields the theorem.

\section{Example}

Using Corollary 1 above, we will find an asymptotic formula for the coefficients of the following bivariate generating function:

$$
F(x, y)=\frac{1-x(1+y)}{\sqrt{1-2 x(1+y)-x^{2}(1-y)^{2}}}
$$

This generating function describes the stationary distributions of a red/blue color-swapping algorithm on the complete graphs $K_{r}$, where the coefficient of $x^{r} y^{s}$ is proportional to the probability that $s$ of the vertices of $K_{r}$ are blue in the stationary distribution, rescaled by a factor proportional to $\left(\begin{array}{c}2 r-2 \\ r\end{array}\right)$. For more details, see [BCCG15]. Because each $y$ term is attached to an $x$ of equal or greater power, the power series expansion of $F$ will have no terms where the power of $y$ is larger than the power of $x$. Thus, we will look at the asymptotics only the case where $\mu:=\lambda^{-1}=\frac{s}{r} \in(0,1)$. (We switch to $\mu$ so that the range of possible directions is bounded.)

The following paragraphs briefly describe how to check the conditions of Corollary 1 computationally. A Maple worksheet providing the code for these computations is available online:

$$
\text { http://people.math.gatech.edu/ tgreenwood3/FPSAC.html }
$$

To begin, we find the critical points of the denominator, $H(x, y)=1-2 x(1+y)-x^{2}(1-y)^{2}$. We can use a Gröbner basis to compute these points in terms of $\mu$. Here, the first polynomial in the basis is as follows:

$$
1-2 \mu+\mu^{2}+\left(-4-2 \mu^{2}+6 \mu\right) x+2 x^{3}+\left(2 \mu^{2}-4 \mu+3\right) x^{2}
$$

Because this is a degree 3 polynomial in $x$, we can solve for the three values of $x$ explicitly in terms of $\mu$. Once the $x$ solutions are found, they can be plugged into the second basis element of the Gröbner basis to compute the corresponding $y$ solutions in terms of $\mu$. We must check that all of these critical points are smooth, that neither $x$ nor $y$ is zero, and that $M$ is not zero at each critical point where $\mu \in(0,1)$. Each of these facts can be verified with additional Gröbner bases. Showing that $M$ is nonzero requires a slightly more complicated Gröbner basis, but it is easy to verify via Sturm sequences that $M$ is never zero for $\mu \in(0,1)$. Finally, checking the critical points for minimality is computationally difficult, but can be achieved for any particular $\mu$ through quantifier elimination, using Mohab Safey El Din's RAGlib package, [Saf15], which relies on the FGb Maple package, [Fau10]. To see how closely the formula approximates coefficients, we look at the case where $\mu=\frac{1}{2}$. Using the Gröbner bases mentioned above, we easily compute the unique minimal critical point, $(p, q)=\left(\frac{1}{4}, 1\right)$. From here, we can compute the following:

$$
\chi_{1}=\frac{1}{8}, \chi_{2}=-\frac{3}{64}, H_{x}\left(\frac{1}{4}, 1\right)=-4, \quad M=-\frac{3}{8}
$$


Thus, from Corollary 1 above (with $\beta=\frac{1}{2}$ ), we have that as $r, s \rightarrow \infty$ with $2=\frac{r+O(1)}{s}$ as $r, s \rightarrow \infty$,

$$
\left[x^{r} y^{s}\right] H(x, y)^{-1 / 2} \sim \frac{r^{-1}\left(\frac{1}{4}\right)^{-r}}{\Gamma\left(\frac{1}{2}\right) \sqrt{\frac{3 \pi}{4}}}=\frac{2 \cdot 4^{r}}{r \pi \sqrt{3}}
$$

If the numerator of $F$ was a monomial $a x^{m} y^{n}$, it would simply shift the terms in the series of $F$ by $m$ in the $x$ variable and $n$ in the $y$ variable, and multiply all the coefficients by $a$. We can break up the numerator of $F$ linearly and compute these shifts separately. Equivalently, to account for the fact that the numerator $G(x, y):=1-x(1+y)$ is not a monomial, we multiply our approximation above by $G$ evaluated at the critical point. In this case, since $G\left(\frac{1}{4}, 1\right)=\frac{1}{2}$, the final approximation is:

$$
\left[x^{r} y^{s}\right] F(x, y) \sim \frac{4^{r}}{r \pi \sqrt{3}}
$$

When $r=70$, this formula gives approximately $3.65924 \cdot 10^{39}$. The actual value of $\left[x^{70} y^{35}\right] F(x, y)$ is approximately $3.59821 \cdot 10^{39}$. The ratio of these values is 1.017 , showing that the approximation is already quite good for $r=70$.

\section{Future Research}

Possible future research directions include finding more complete asymptotic expansions for the coefficients of $H^{-\beta}$, and also extending to more variables. Finding a more complete asymptotic expansion should be possible by analyzing the Cauchy integral using the same contour, but with more precise error handling. Extending the formula to more variables is challenging because the change of variables needed to approximate a multivariate function by a univariate linear function quickly becomes complicated. Another research direction would be to look at other types of algebraic singularities. For example, one could study the coefficients of a function $F(x, y)$ which is known to satisfy some polynomial equation but may not have the form $H^{-\beta}$.

Combining these results with other asymptotic techniques may yield stronger results and more complete asymptotic expansions, too. For example, creative telescoping methods take the generating function in question and find a partial differential equation that the function satisfies. By finding a basis of solutions to this differential equation, one can find complete asymptotic expansions to the coefficients of the generating function. Unfortunately, it is often difficult to find the correct coefficients of the solution to the PDE this is referred to as the connection problem. However, if the leading-term asymptotics of the solution are known, the connection problem can often be solved. Thus, combining these creative telescoping methods with the first-order asymptotics results in this paper, one may be able to analyze generating functions without too many technical computations.

\section{Acknowledgements}

The University of Pennsylvania supported this research in part, as I completed my doctoral thesis. I thank my advisor, Robin Pemantle, for his generous help and guidance. Mohab Safey El Din and Rainer Sinn graciously helped significantly with computations in the example. Thank you to the referees for their valuable feedback and references. 


\section{References}

[BCCG15] S. Butler, F. Chung, J. Cummings, and R. Graham. Edge flipping in the complete graph. Preprint. Available online: http://www.math.ucsd.edu/ ronspubs/pre_ flipping.pdf

[BR83] E.A. Bender and L.B. Richmond. Central and local limit theorems applied to asymptotic enumeration. II. Multivariate generating functions. In J. Combin. Theory Ser. A, vol 34 (1983) pp. 255-265.

[Fau10] J.-C. Faugère. FGb: A Library for Computing Gröbner Bases. In Komei Fukuda, Joris Hoeven, Michael Joswig, and Nobuki Takayama, editors, Mathematical Software ICMS 2010, vol 6327 of Lecture Notes in Computer Science, pp. 84-87, Berlin, Heidelberg, September 2010. Available online: http://www-polsys.lip6.fr/ jcf/FGb/FGb/index.html

[FO90] P. Flajolet and A. M. Odlyzko. Singularity analysis of generating functions. In SIAM J. Discrete Math., vol 3 (1990) pp. 216-240. Available online: http://algo.inria.fr/ flajolet/Publications/Flod90b.pdf

[GR92] Z. Gao and L.B. Richmond. Central and local limit theorems applied to asymptotic enumeration. IV. Multivariate generating functions. In J. Comput. Appl. Math., vol 41 (1992) pp. 177-186.

[Hwa96] Hwang, H.-K. Large deviations for combinatorial distributions. I. Central limit theorems. In Ann. Appl. Probab., vol 6 (1996) pp. 297-319. Available online: http:// projecteuclid.org/download/pdf_1/euclid.aoap/1034968075

[Hwa98] Hwang, H.-K. Large deviations for combinatorial distributions. II. Local limit theorems. In Ann. Appl. Probab., vol 8 (1998) pp. 163-181. Available online: http:// projecteuclid.org/download/pdf_1/euclid.aoap/1027961038

[PW13] R. Pemantle and M. Wilson. Analytic Combinatorics in Several Variables, Cambridge University Press, New York, 2013. Available online: http://www.math.upenn.edu/ pemantle/ACSV.pdf

[RW08] A. Raichev and M. Wilson. Asymptotics of Coefficients of Multivariate Generating Functions: Improvements for Smooth Points. In Electron. J. Combin., vol 15 (2008). Available online: http://www.combinatorics.org/ojs/index.php/eljc/article/ view/v15i1r89/pdf

[Saf15] M. Safey El Din. RAGlib, a Maple package dedicated to real solutions of polynomial systems, March 2015. Available online: http://www-polsys.lip6.fr/ safey/RAGLib/ 